\title{
SERUM LIPOPOLYSACCHARIDE BINDING PROTEIN AS A PREDICTOR AND PROGNOSTIC BIOMARKER FOR CIRRHOSIS RELATED ACUTE KIDNEY INJURY
}

Hesham Kamal ElSayegh ${ }^{1}$, Neveen Lewis Mikhael Saad ${ }^{2}$, Hesham Abdallah Mohamed Elghoneimy' ${ }^{1}$ Mohamed Adel Abd El Aziz ${ }^{3}$, Walaa Ahmed Mohamed Eidresha ${ }^{1}$

${ }^{1}$ Department of Internal Medicine, ${ }^{2}$ Department of Clinical and Chemical Pathology, ${ }^{3}$ Department of Tropical Medicine, Faculty of Medicine, University of Alexandria, Egypt

\begin{tabular}{|l|}
\hline INTRODUCTION \\
-Acute kidney injury (AKI), defined as an increase in serum creatinine $\geq 0.3 \mathrm{mg} / \mathrm{dL}$ ( 26.5 \\
$\mu$ moL/L) $\leq 48$ hours, or a $50 \%$ increase from baseline, is one of the most serious \\
complications of liver cirrhosis. It occurs in about $19 \%$ of hospitalized cases with liver \\
cirrhosis and it carries a bad prognosis. The acute kidney dysfunction is associated with \\
mortality in cases with cirrhosis. Moreover, it usually occurs concomitantly with other \\
cirrosis related complications like spontaneous bacterial peritonitis (SBP) and bleeding \\
varices. \\
- Multiple factors could increase the risk for this complication including infections, \\
hypovolemia, use of vasodilators, and non-steroidal anti-inflammatory drugs. Because of \\
its unfavorable prognosis, potential triggers for AKI should be identified and removed in \\
such cases. This includes correction of hypovolemia, discontinuation of nephrotoxic \\
drugs, eradication of infections and management of other complications like variceal \\
bleeding. \\
•Lipopolysaccharide binding protein (LBP) is an acute phase protein that is synthesized \\
by the liver. It is secreted into the blood stream in response to infection with Gram- \\
negative bacteria. Recent studies have reported that LBP is an important mediator of \\
AKI in critically ill patients. However, after intensive online research, the current \\
literature had poor data regarding the role of that biomarker in AKI in cirrhotic patients. \\
\hline
\end{tabular}

AIM OF THE WORK

- This study was conducted to study the serum level of LBP in patients having prognostic value of LBP in relation to renal outcomes along with all-cause mortality

\section{PATIENTS \& METHODS}

This prospective study included 80 patients with decompensated cirrhosis (jaundice, encephalopathy, coagulopathy or variceal hemorrhage). They were divided into two groups, Group I (40 cases - no AKI) and Group II (40 cases - AKI). Patients with kidney disease were excluded

- The included cases were subjected to full history taking and thorough clinical -

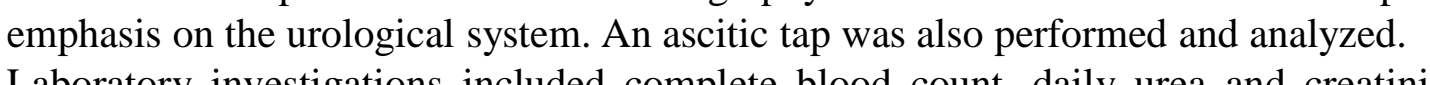

Laboratory investigations included complete blood count, daily urea and creatinine,
hepatological viral markers. Regarding LBP assessment, it was measured by ELISA within 24 hours from admission in Group I, and within 24 hours of AKI detection in Group II.

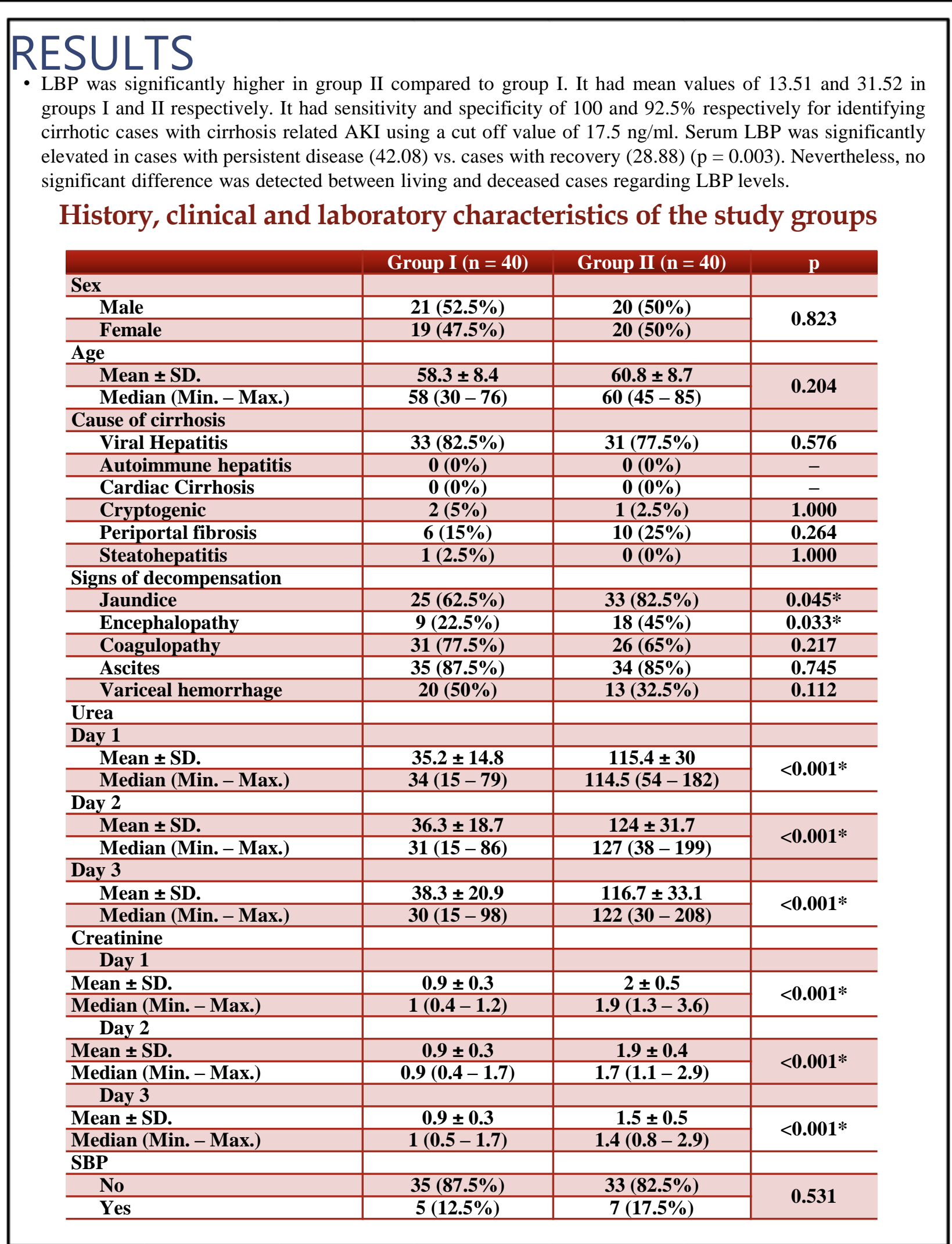

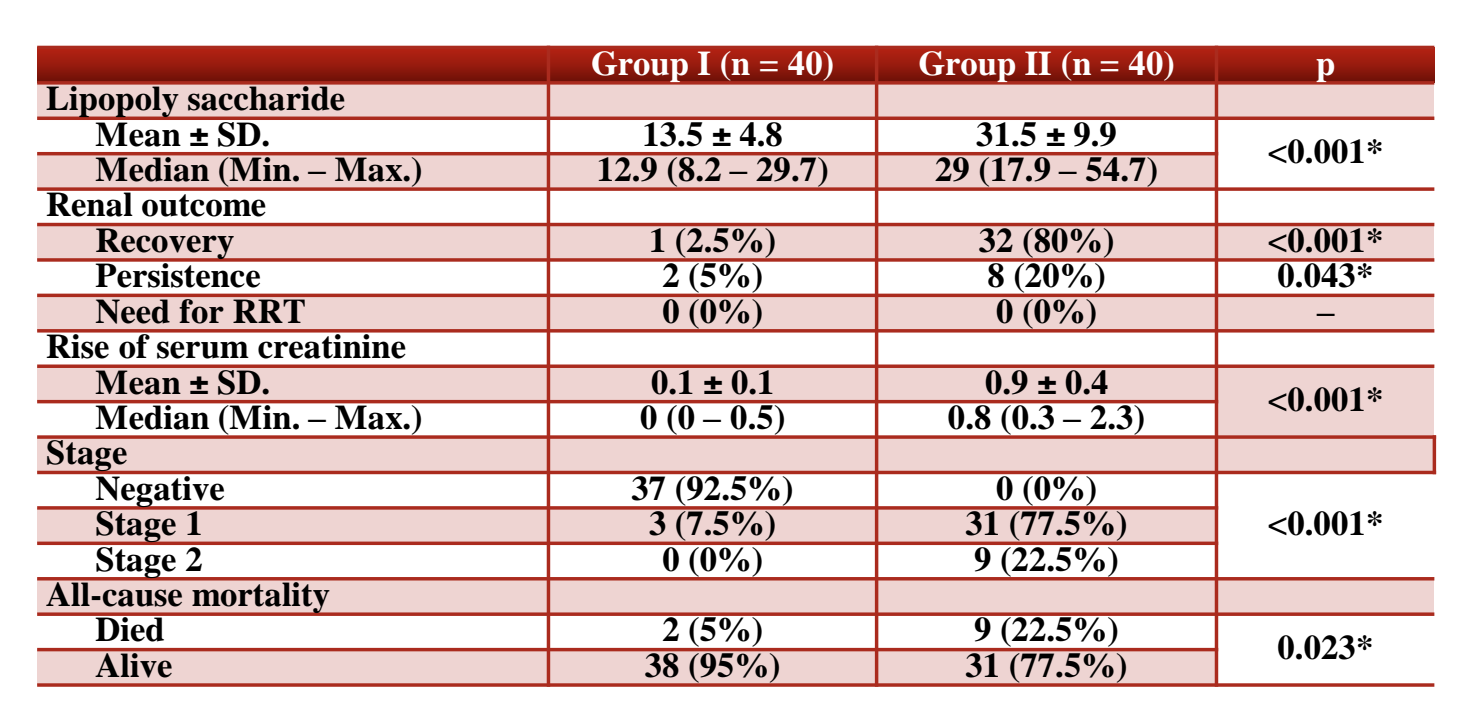

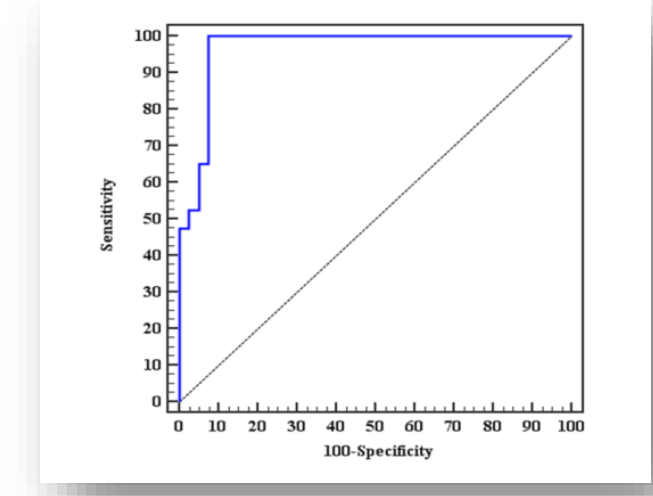

ROC curve for LBP to diagnose patients with cirrhosis related AKI

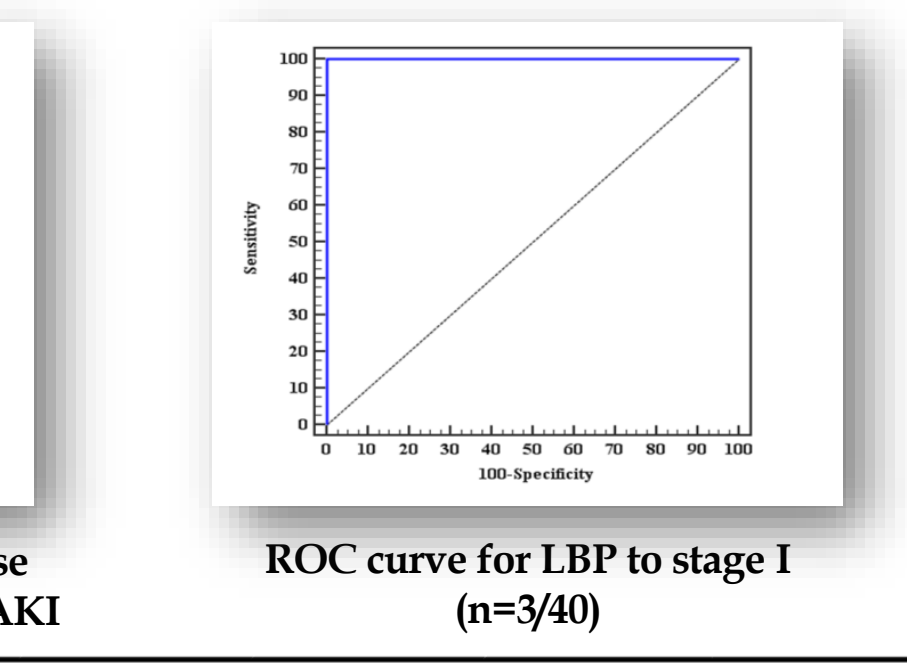

CONCLUSIONS

- Based on the previous findings, LBP appears to be a sensitive biomarker for the diagnosis of AKI in cirrhotic patients. Besides, higher levels are associated with an increased risk of persistent renal disease.

$$
\begin{gathered}
\text { 20210Alexandria Faculty of Medicine } \\
\text { CC-BY-NC }
\end{gathered}
$$

MEDICINE $\mid$ PACU⿴囗十) 